\title{
A Hybrid Deep Learning Approach for Diagnosis of the Erythemato-Squamous Disease
}

\author{
Sayan Putatunda ${ }^{\mathrm{a}}$ \\ ${ }^{a}$ Indian Institute of Management Ahmedabad, India
}

\begin{abstract}
The diagnosis of the Erythemato-squamous disease (ESD) is accepted as a difficult problem in dermatology. ESD is a form of skin disease. It generally causes redness of the skin and also may cause loss of skin. They are generally due to genetic or environmental factors. ESD comprises six classes of skin conditions namely, pityriasis rubra pilaris, lichen planus, chronic dermatitis, psoriasis, seboreic dermatitis and pityriasis rosea. The automated diagnosis of ESD can help doctors and dermatologists in reducing the efforts from their end and in taking faster decisions for treatment. The literature is replete with works that used conventional machine learning methods for the diagnosis of ESD. However, there isn't much instances of application of Deep learning for the diagnosis of ESD.

In this paper, we propose a novel hybrid deep learning approach i.e. Derm2Vec for the diagnosis of the ESD. Derm2Vec is a hybrid deep learning model that consists of both Autoencoders and Deep Neural Networks. We also apply a conventional Deep Neural Network (DNN) for the classification of ESD. We apply both Derm2Vec and DNN along with other traditional machine learning methods on a real world dermatology dataset. The Derm2Vec method is found to be the best performer (when taking the prediction accuracy into account) followed by DNN and Extreme Gradient Boosting.The mean CV score of Derm2Vec, DNN and Extreme Gradient Boosting are $96.92 \%, 96.65 \%$ and $95.80 \%$ respectively.
\end{abstract}

Keywords: Autoencoders, Deep Learning, Dermatology, Erythemato-Squamous Disease, Machine Learning, Medical Informatics

\section{Introduction}

Erythemato-squamous disease (ESD) is a form of skin disease. It generally causes redness of the skin and also may cause loss of skin. ESDs are generally due to genetic or environmental factors [1]. ESD comprises six classes of skin conditions namely,

\footnotetext{
Pre-review version of the paper accepted at the 2020 IEEE CONECCT Conference. 978-1-7281-6828-9/20/\$31.00 (C) 2020 IEEE

Email address: sayanp@iima.ac.in (Sayan Putatunda )
} 
pityriasis rubra pilaris, lichen planus, chronic dermatitis, psoriasis, seboreic dermatitis and pityriasis rosea. However, the diagnosis of ESD is accepted as a difficult problem in Dermatology. The reason why ESD is difficult to diagnose is due to the fact that these diseases share many clinical and histopathological attributes with erythema and scaling. Another reason is that one disease may show the symptoms of another disease at the initial stages [2]. Thus, a detailed observation skills and high experience are required from physicians to evaluate both clinical and histopathological features to correctly diagnose ESD [3]. So, the automated diagnosis of ESD can help doctors and dermatologists in reducing the efforts from their end and in taking faster decisions for treatment.

In the literature, there are a quite a few instances of works that proposed various machine learning methods such as decision trees, support vector machines, artificial neural networks and more for automated detection of the type of Erythemato-squamous disease [3]. We will discuss in detail about these works in Section 2. In recent years, with the rise in computing power and availability of cheap memory devices along with cloud computing, deep learning has been very successful in many fields such as natural language processing [4], biomedicine [5], computer vision [6] and more.

The main contribution of this paper is in the development of a novel hybrid deep learning approach i.e. Derm2 Vec for the diagnosis of the Erythemato-Squamous disease (ESD) that hasn't been reported in the literature earlier to the best of our knowledge. Derm2 Vec is a hybrid deep learning approach that comprises both Autoencoders and Deep Neural Networks. Also, we find that there haven't been many works reported in the literature regarding the applications of deep neural networks for the classification of ESD. Although the literature is replete with works that used conventional machine learning methods (such as Random forests, artificial neural netwroks, Extreme Gradient Boosting, K-nearest neighbors, decision trees, support vector machines and more) for the diagnosis of ESD. In this paper, we apply both Derm2Vec and DNN (after tuning the hyperparameters) along with other conventional machine learning methods on a real world dermatology dataset. The Derm 2 Vec method is found to be the best performer when taking the prediction accuracy into account.

The rest of this paper is organized as follows. In Section 2, we present a brief review of the literature. In Section 3 , we describe the dataset used in this paper. This is followed by Sections 4 and 5 where we describe our proposed methodology and the experimental results respectively. Finally, Section 6 concludes the paper.

\section{Related Work}

There have been many works in the medical informatics literature on the applications of machine learning and expert systems and how it complements physicians/practitioners in decision making. For example, Cruz \& Wishart [7] used machine learning for cancer prediction. Random forests was used for classification of the Alzheimer's disease [8]. Deep learning was used for the classification of self-care problems in children with physical disabilities [9]. There have been many applications of machine learning for glaucoma screening [10], retinal hemorrhage detection [11], lymphoma classification [12] and many more. 
Automated classification of the type of Erythemato-squamous disease using machine learning and expert systems is reported in the literature. The first such work is that of Demiroz et al. [2] where the authors developed a new classifier called "Voting feature intervals-5" for the differential diagnosis of ESD. Guvenir \& Emeksiz [13] used three classification algorithms namely, Voting feature intervals-5, Naïve Bayes and nearest neighbor classification for diagnosis of the type of ESD. Ubeyli [14] used multi layer perceptron neural networks and Xie \& Wang [15] used support vector machines for the classification of ESD. Even tree based methods such as CHAID decision trees [1] and ensemble of decision trees [3] have been used for analysis and diagnosis of ESD. Nanni [16] used an ensemble of support vector machines on random subspace and Menai [17] applied random forests for the diagnosis of ESD.

Some of the other interesting methods reported in the literature for the diagnosis of ESD are fuzzy classification [18], neuro-fuzzy inference systems [19], k-means clustering [20], boosting [21] and genetic programming [22]. We find that none of the past works reported in the literature have used Deep learning to the best of our knowledge. In this paper, we use Deep neural networks for the differential diagnosis of ESD and propose a novel hybrid deep learning method i.e. Derm2Vec (see Section 4.3).

\section{Dermatology Data}

In this paper, we use the dermatology dataset that was first used by Ubeyli \& Guler [19] where the aim was to determine the type of ESD. This dataset is publicly available in the UCI machine learning repository [23]. The dataset contains 33 attributes/predictor variables where 12 are for clinical attributes (namely, (a) erythema, (b) scaling, (c) definite borders, (d) itching, (e) koebner phenomenon, (f) polygonal papules, (g) follicular papules, (h) oral mucosal involvement, (i) knee and elbow involvement, (j) scalp involvement, (k) family history and (l) Age).

The remaining 21 features are for the histopathological attributes (namely, (a) melanin incontinence, (b) eosinophils in the infiltrate, (c) PNL infiltrate, (d) fibrosis of the papillary dermis, (e) exocytosis, (f) acanthosis, (g) hyperkeratosis, (h) parakeratosis, (i) clubbing of the rete ridges, $(j)$ elongation of the rete ridges, $(\mathrm{k})$ thinning of the suprapapillary epidermis, (l) spongiform pustule, (m) munro microabcess, (n) focal hypergranulosis, (o) disappearance of the granular layer, (p) vacuolisation and damage of basal layer, (q) spongiosis, saw-tooth appearance of retes, (r) follicular horn plug, (s) perifollicular parakeratosis, $(\mathrm{t})$ inflammatory monoluclear inflitrate and $(\mathrm{u})$ band-like infiltrate). The total number of features after performing one-hot encoding for the categorical variables becomes 129 .

The total number of observations in the dataset are 366. However, there are around 8 missing values for the "Age" variable in the dataset and we won't be considering these

observations in our analysis. So, finally the dataset has 358 instances after removing missing values. The target variables had 6 classes and the number of instances in each are- (a) psoriasis- 111, (b) seboreic dermatitis- 60, (c) lichen planus- 71, (d) pityriasis rosea- 48, (e) chronic dermatitis- 48 and (f) pityriasis rubra pilaris- 20. 


\section{Methodology}

Sections 4.1 and 4.2 give a brief overview of artifical neural networks and deep learning respectively. In Section 4.3, we discuss our proposed method i.e. Derm2Vec and Section 4.4 metions the different machine learning methods that we will use for comparison of performance in this paper.

\subsection{Artificial Neural Networks}

To understand Deep learning (discussed in Section 4.2), first we need to understand Artifical Neural Networks (ANNs). The origin of ANNs can be traced to the study of information processing in life sciences [24-26]. McCulloch \& Pitts [24] worked on developing nets of simple logical operators to model biological systems. Rosenblatt [27] introduced the concept of "perceptron" that is a biologically inspired learning algorithm. Neural networks are used for various statistical modeling and data analysis tasks and it is seen as an alternative to non-linear regression [28].

In this paper, we focus on the "feed-forward neural networks". Bishop [29] describes a two layered feed-forward neural network architecture consisting of an input layer that is followed by a hidden layer (that consists of hidden nodes) and finally, an output layer. The hidden nodes are like processing units that contains activation functions. Some of the commonly used activation functions are Sigmoid, ReLu, tanh and more [30]. A feed forward neural networks performs layered computations where the hidden unit activations are computed using the input layer and then the output is calculated using the hidden unit activations [31]. Please refer to Bishop [29] for more details on the modus-operandi of the feed-forward neural networks.

Nowadays, artificial neural networks are widely used in various applications such as weather forecasting [32], clinical medicine [33], Forex prediction [34], Location/Travel time prediction for GPS Taxis [35-37] and more. Please see Bishop [38] for more details on artificial neural networks.

\subsection{Deep learning: Deep Neural Networks and Autoencoders}

Goodfellow et al. [39] describes deep learning as a subset of machine learning and as a form of "representation learning". Here the focus is on using the raw data to extract high level features. Chollet [40] describes deep learning as learning from successive layers, each layer being some meaningful representation. In the recent years, deep learning has tasted success in various applications such as natural language processing [4], biomedicine [5], computer vision [6] and more. There are different kinds of deep learning architectures such as Recurrent neural networks and Convolutional neural networks (see Goodfellow et al. [39] for more details on different deep learning techniques). However, in this paper we will focus on Deep Neural Networks (DNNs) and Autoencoders.

Deep neural networks (DNNs) originated from Artificial neural networks (see Section 4.1). ANNs with many hidden layers is known as DNNs [5]. These number of hidden layers determine the "depth" of a DNN [40]. An Autoencoder is a type of deep learning method where the input and the output are same. It is classified as selfsupervised learning method by Chollet [40]. An Autoencoder consists of two functions namely, (a) Encoder function- here the raw input data is converted into representations 
and (b) Decoder function- here the representations from the encoder layer are converted back to the input data. The goal of an autoencoder is to preserve as much information as possible and also add new representations on top of the raw input data [39]. Some of the great applications of Autoencoders include dimensionality reduction [41], cyberemphatic design [42], molecular design [43] and many more.

\subsection{Proposed Method: Derm2Vec}

In this paper, first we apply a conventional DNN on the dataset for the prediction of the Erythemato-Squamous disease. The usage of DNN has't been reported in the literature (see Section 2) for the diagnosis of ESD. Although ANNs have been used earlier as mentioned in Section 2 .

In this paper, we propose a novel hybrid deep learning approach that is a two-step modeling approach comprising an autoencoders and a DNN for multi-class classification of type of ESD. This hasn't been reported (to the best of our knowledge) in the dermatology informatics literature. We will refer to our proposed method as "Derm2Vec". Figure 1 shows the modus-operandi of the Derm2Vec method.

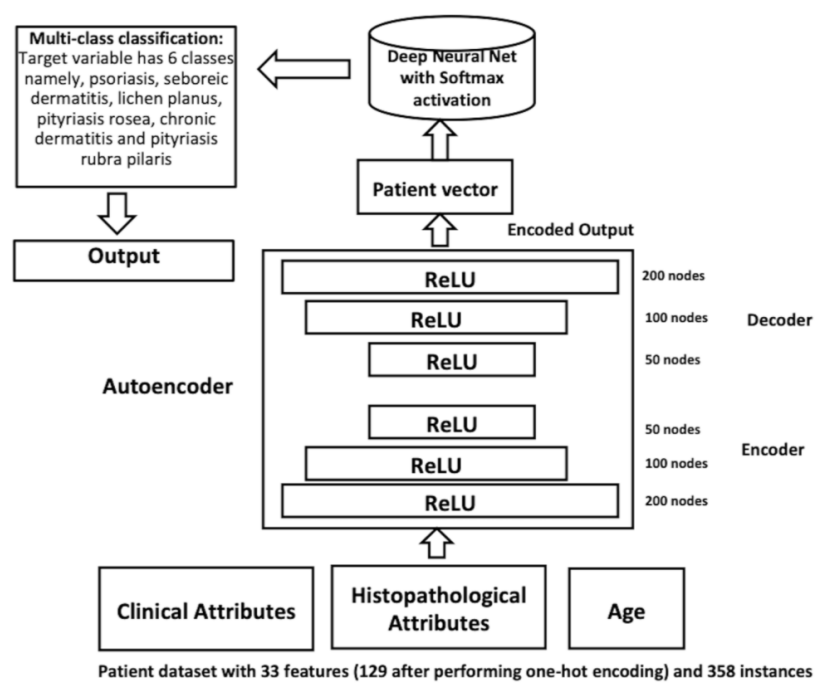

Figure 1: The Derm2Vec method

We can see in Figure 1 that high dimensional the input data (i.e. 129 features related to clinical and Histopathological attributes along with Age) is passed through an Autoencoder that comprises three encoder and three decoder layers containing 50, 100 and 200 nodes in each. The different encoding dimensions used by the encoder are $4,8,16,32,40,48$ and more (see Section 5.2). The values from the innermost encoder layer is taken i.e. the Encoded output that represents a dense patient vector. We then apply a DNN (comprising a single hidden layer with 100 nodes or two hidden layers with 100 nodes in each) on the patient vector to get the predicted output. Since, the target variable contains 6 classes i.e. this is a multi-class classification problem, so we use the "Softmax" activation function in the output layer of the DNN. 


\subsection{Other Methods}

In this paper, we will compare the performance of both our proposed method i.e. Derm2 Vec and a conventional DNN method with other conventional machine learning techniques that have been used for the prediction of the Erythemato-Squamous disease in the literature as discussed in Section 2. Some of the techniques we will use in this paper for comparison are Decision trees [44, 45], Artificial Neural Networks (see Section 4.1], ensemble learning methods such as Extreme Gradient Boosting [46] and Random Forests [47], K nearest neighbors [48], Support Vector Classification [49] and finally, the Gaussian Naïve Bayes [50].

Please refer to [29], [51], [52] and [30] for a detailed account on the various machine learning methods mentioned above.

\section{Experimental Results}

Our goal is to predict the type of Erythemato-Squamous disease using our proposed method i.e. Derm2Vec along with a Deep Neural Network on the dermatology dataset described in Section 3 This is a multi-class classification problem as the target variable has 6 classes. We will compare the performance of the above mentioned method with other conventional machine learning techniques that have been used in the literature for the prediction of ESD (see Section 2) and also mentioned in Section 4.4 such as Extreme Gradient Boosting (XGBoost), Artificial Neural Network (ANN), Random Forest (RF), Decision Tree (DT), Naïve Bayes (NB), K-nearest neighbors (KNN) and Support Vector Classification (SVC).

All the experiments are conducted in a system with mac OSX, 16 GB RAM and Intel core $i 7$ processor. The data analysis and model development were done in Python [53]. We use the scikit-learn [54], Tensorflow [55] and Keras [56] libraries for implementing the various machine learning and deep learning techniques used in this paper.

\subsection{Evaluation Metrics}

We apply the above mentioned methods on the dermatology dataset and perform k-fold cross-validation [57]. In this paper, we perform 10-fold cross-validation i.e. the dataset is partitioned into 10 equal sets or folds and then 10 subsequent iterations are performed where 9 folds are used for model training and 1 fold is withheld for validation. We use the Mean cross-validation score i.e. the Mean CV score as a evaluation metric for our experiments in this paper (see Section 5.2). The Mean CV score is the average of the accuracy scores obtained in each iterations while performing the 10 fold cross-validation.

A higher value of mean CV score indicates better performance of the method. It is reported in terms of percentage (\%) in this paper.

\subsection{Results: Classification of the Erythemato-Squamous Disease}

Table 1 describes the mean CV scores when we apply Deep Neural Network (DNN) with different hyperparamters on the dataset and perform 10-fold cross validation. We run multiple iterations of DNN with different number of hidden layers such as 1,2 or 3 containing different hidden nodes such as 100, 200 and 300. Since this is in a 
multi-class classification setting, so the output layer of the DNN will have a Softmax activation function. We also use "Dropouts" that ensure that the deep neural network model doesn't overfits [58]. Here, Dropout $=0.5$ means that $50 \%$ of the units are dropped randomly during training. We find that the highest mean CV score for DNN (that consists of 1 hidden layer, 100 hidden nodes and dropout $=0.5$ ) is $96.65 \%$.

Table 1: Mean CV score for DNN with different hyperparameters

\begin{tabular}{ccccc}
\hline S1 no. & \multicolumn{3}{c}{ DNN hyperparameters } & Mean CV \\
\cline { 2 - 4 } & $\begin{array}{c}\text { No. of hidden } \\
\text { layers }\end{array}$ & $\begin{array}{c}\text { No. of hidden } \\
\text { nodes }\end{array}$ & Dropouts & \\
\hline 1 & 2 & $(100,100)$ & No & 96.1 \\
\hline 2 & 1 & 100 & No & 95.8 \\
\hline 3 & 2 & $(100,100)$ & Yes $(0.5)$ & 96.37 \\
\hline 4 & 1 & 100 & Yes $(0.5)$ & 96.65 \\
\hline 5 & 3 & $(100,100,100)$ & No & 96.37 \\
\hline 6 & 3 & $(100,100,100)$ & Yes $(0.5)$ & 96.08 \\
\hline 7 & 1 & 200 & No & 95.8 \\
\hline 8 & 1 & 200 & Yes $(0.5)$ & 96.09 \\
\hline 9 & 1 & 300 & No & 95.81 \\
\hline 10 & 1 & 300 & Yes $(0.5)$ & 95.25 \\
\hline
\end{tabular}

We now apply the Derm2Vec method on the dermatology dataset with different hyperparameters and run multiple iterations as described in Table 2 Here we tune the hyperparameters of the Autoencoder and the DNN. For the Autoencoder, the encoder and decoder comprises 3 layers each with 50, 100 and 200 nodes as shown in Figure 1. The only hyperparameter we tune is the encoding dimensions i.e. we compress the high dimensional dataset (i.e. containing 129 features) into a low dimensional space. We vary the encoding dimensions from $4,8,16,32,40,48, \ldots$, upto 88 as described in Table 2 We also tune the hyperparameters of the subsequent DNN of Derm2Vec i.e. Dropouts, the number of hidden layers and the number of hidden nodes. We find that the highest mean CV score is $96.92 \%$ that is higher than what we got for DNN in Table 1. Thus, Derm2Vec performs better than DNN. In fact from Tables 1 and tab2 we can clearly see that the best performing configuration of Derm2Vec i.e. DNN with 1 hidden layer, 100 hidden nodes and dropout $=0.5$ when complemented with an Autoencoder with encoding dimension of 32 perform better than the stand alone DNN with similar configuration (i.e. 1 hidden layer, 100 hidden nodes and dropout $=0.5$ ). This shows that the proposed hybrid deep neural network approach i.e. Derm2Vec is a better performer (when taking the prediction accuracy into consideration) than a conventional deep neural network.

In Table 3, we compare the performance of our proposed Derm2Vec method along with the DNN with some of the other conventional machine learning methods used in the literature for the diagnosis of ESD (see Sections 2 and 4.4). We compare Derm2Vec and DNN with other methods such as Extreme Gradient Boosting (XGBoost), Random Forest (RF), Decision Tree (DT), Naïve Bayes (NB), Artificial Neural Network (ANN), K-nearest neighbors (KNN) and Support Vector Classification (SVC). For ANN, we use a simple architecture comprising one hidden layer with two hidden nodes. As far as the choice of kernel for the SVC method is concerned, we chose the "Radial basis 
Table 2: Mean CV score for Derm2Vec with different hyperparameters

\begin{tabular}{cccccc}
\hline \multirow{2}{*}{ Sl no. } & $\begin{array}{c}\text { Autoencoder parameter- } \\
\text { Encoding dimensions }\end{array}$ & \multicolumn{3}{c}{ DNN hyperparameters } & Mean CV \\
\cline { 3 - 5 } & & $\begin{array}{c}\text { No. of hidden } \\
\text { layers }\end{array}$ & $\begin{array}{c}\text { No. of hidden } \\
\text { nodes }\end{array}$ & Dropouts & \\
\hline 1 & 4 & 1 & 100 & Yes $(0.5)$ & 95.52 \\
\hline 2 & 8 & 1 & 100 & Yes $(0.5)$ & 96.37 \\
\hline 3 & 16 & 1 & 100 & Yes $(0.5)$ & 95.79 \\
\hline 4 & 24 & 1 & 100 & Yes $(0.5)$ & 96.35 \\
\hline 5 & 32 & 1 & 100 & Yes $(0.5)$ & 96.92 \\
\hline 6 & 40 & 1 & 100 & Yes $(0.5)$ & 94.96 \\
\hline 7 & 48 & 1 & 100 & Yes $(0.5)$ & 96.37 \\
\hline 8 & 32 & 2 & 100 & No & 94.97 \\
\hline 9 & 32 & 2 & 100 & Yes $(0.5)$ & 96.92 \\
\hline 10 & 32 & 1 & 100 & Yes $(0.5)$ & 96.1 \\
\hline 11 & 56 & 1 & 100 & Yes $(0.5)$ & 96.94 \\
\hline 12 & 64 & 1 & 100 & Yes $(0.5)$ & 95.52 \\
\hline 13 & 72 & 1 & 100 & Yes $(0.5)$ & 95.53 \\
\hline 14 & 80 & 1 & 100 & Yes $(0.5)$ & 96.92 \\
\hline 15 & 88 & 1 & 100 & Yes $(0.5)$ & 96.36 \\
\hline
\end{tabular}

function (RBF)" [49]. For the KNN method, we use $K=5$. The different hyperparamters that we chose for Random forests are $n_{-}$estimators $=100$ and max_depth $=$ 3. Similarly, for XGBoost the hyperparameters selected were learning_rate $=0.05$, n_estimators $=300$ and max_depth $=3$.

Table 3: Comparing Derm2Vec and DNN with other methods such as Extreme Gradient Boosting (XGBoost), Random Forest (RF), Decision Tree (DT), Naïve Bayes (NB), Artificial Neural Network (ANN), $\mathrm{K}$-nearest neighbors (KNN) and Support Vector Classification (SVC)

\begin{tabular}{lc}
\hline Method & $\begin{array}{c}\text { Mean CV } \\
\text { score (in \%) }\end{array}$ \\
\hline Derm2Vec & 96.92 \\
\hline DNN & 96.65 \\
\hline XGBoost & 95.80 \\
\hline DT & 93.10 \\
\hline ANN* & 74.29 \\
\hline SVC** & 82.13 \\
\hline RF & 51.13 \\
\hline NB & 92.68 \\
\hline KNN*** & 79.30 \\
\hline$*$ ANN has 1 hidden layer with 2 \\
hidden nodes \\
** SVC has RBF kernel \\
${ }^{* * *}$ K=5 in KNN
\end{tabular}

The Derm2Vec method is found to be the best performer (when taking the prediction accuracy into account) followed by DNN and Extreme Gradient Boosting as described in Table 3 . Both Derm2Vec and DNN perform better than XGBoost, RF, DT, ANN, SVC, NB and KNN. The mean CV score of Derm2Vec and DNN are $96.92 \%$ and 96.65\%. However, the mean CV score of XGBoost, DT, ANN, SVC, RF, NB and KNN are $95.80 \%, 93.10 \%, 74.29 \%, 82.13 \%, 51.13 \%, 92.68 \%$ and $79.30 \%$ respectively. 


\section{Conclusion}

In this paper, we propose a novel hybrid deep learning approach i.e. Derm2Vec for the diagnosis of the Erythemato-Squamous disease (ESD) that to the best of our knowledge, hasn't been reported in the literature. Also, we find that there haven't been many works reported in the literature regarding the applications of deep neural networks for the classification of ESD. Although the literature is replete with works that used conventional machine learning methods (namely, Random forests, artificial neural networks, Extreme Gradient Boosting, K-nearest neighbors, decision trees, support vector machines and Naïve Bayes) for the diagnosis of ESD.

We apply both Derm2Vec and a Deep Neural Network (after tuning the hyperparameters) along with other conventional machine learning methods as mentioned above on a real world dermatology dataset. The Derm2Vec method is found to be the best performer when taking the prediction accuracy into account. Thus, we conclude that our proposed hybrid deep learning approach i.e. Derm2Vec is an effective method for the diagnosis of ESD. We feel that our proposed hybrid deep learning method Derm2Vec can be extended with some modifications in other areas of medicine such as diagnosis of liver disease, cancer prediction, prediction of diabetes and more. We plan to work in this direction in the future.

\section{References}

\section{References}

[1] A. M. Elsayad, M. Al-Dhaifallah, A. M. Nassef, Analysis and Diagnosis of Erythemato-Squamous Diseases Using CHAID Decision Trees, in: 15th International Multi-Conference on Systems, Signals and Devices (SSD), IEEE, 2018. doi $10.1109 /$ SSD . 2018.8570553.

[2] G. Demiroz, H. A. Govenir, N. Ilter, Learning Differential Diagnosis of Eryhemato-Squamous Diseases using Voting Feature Intervals, Aritificial Intelligence in Medicine 13 (1998) 147-165.

[3] M. E. B. Menai, N. Altayash, Differential Diagnosis of Erythemato-Squamous Diseases Using Ensemble of Decision Trees, in: Modern Advances in Applied Intelligence, 2014, pp. 369-377.

[4] L. Deng, Y. Liu, Deep Learning in Natural Language Processing, 1 ed., Springer, Singapore, 2018. doi $10.1007 / 978-981-10-5209-5$

[5] P. Mamoshina, A. Vieira, E. Putin, A. Zhavoronkov, Applications of deep learning in biomedicine, Mol. Pharmaceutics 13 (2016) 1445-1454.

[6] A. Voulodimos, N. Doulamis, A. Doulamis, E. Protopapadakis, Deep learning for computer vision: A brief review, Computational Intelligence and Neuroscience (2018).

[7] J. Cruz, D. Wishart, Applications of machine learning in cancer prediction and prognosis, Cancer Informat 2 (2006). 
[8] K. R. Gray, P. Aljabar, R. A. Heckemann, A. Hammers, D. Rueckert, Random forest-based similarity measures for multi-modal classification of alzheimer's disease, NeuroImage 65 (2013) 167-175.

[9] S. Putatunda, Care2vec: A deep learning approach for the classification of selfcare problems in physically disabled children, arXiv:1812.00715 [cs.LG], 2018.

[10] J. Cheng, J. Liu, Y. Xu, F. Yin, D. W. K. Wong, N.-M. Tan, D. Tao, C.-Y. Cheng, T. Aung, T. Y. Wong, Superpixel classification based optic disc and optic cup segmentation for glaucoma screening, IEEE Transactions on Medical Imaging 32 (2013) 1019-1032.

[11] L. Tang, M. Niemeijer, J. M. Reinhardt, M. K. Garvin, M. D. Abramoff, Splat feature classification with application to retinal hemorrhage detection in fundus images, IEEE Transactions on Medical Imaging 32 (2013) 364-375.

[12] Y. Luo, A. R. Sohani, E. P. Hochberg, P. Szolovits, Automatic lymphoma classification with sentence subgraph mining from pathology reports, Journal of the American Medical Informatics Association 21 (2014) 824-832.

[13] H. Guvenir, N. Emeksiz, An expert system for the differential diagnosis of erythemato-squamous diseases, Expert Systems with Applications 18 (2000) 4349.

[14] E. D. Ubeyli, Combined neural networks for diagnosis of erythemato-squamous diseases, Expert Systems with Applications 36 (2009) 5107-5112.

[15] J. Xie, C. Wang, Using support vector machines with a novel hybrid feature selection method for diagnosis of erythemato-squamous diseases, Expert Systems with Applications 38 (2011) 5809-5815.

[16] L. Nanni, An ensemble of classifiers for the diagnosis of erythemato-squamous diseases, Neurocomputing 69 (2006) 842-8845.

[17] M. E. B. Menai, Random forests for automatic differential diagnosis of erythemato-squamous diseases, International Journal of Medical Engineering and Informatics 7 (2015).

[18] S. Lekkas, L. Mikhailov, Evolving fuzzy medical diagnosis of pima indians diabetes and of dermatologica diseases, Artificial Intelligence in Medicine 50 (2010) $117-126$.

[19] E. Ubeyli, I. Guler, Automatic detection of erythemato-squamous diseases using adaptive neuro-fuzzy inference systems, Comput. Biol. Med. 35 (2005) 421-433.

[20] E. D. Ubeyli, E. Dogdu, Automatic detection of erythemato-squamous diseases using k-means clustering, Journal of Medical Systems 34 (2010) 179-184.

[21] N. Badrinath, G. Gopinath, K. Ravichandran, Design of automatic detection of erythemato-squamous diseases through threshold-based abc-felm algorithm, Journal of Artificial Intelligence 6 (2013) 245-256. 
[22] C. C. Bojarczuk, H. S. Lopes, A. A. Freitas, Data mining with constrainedsyntax genetic programming: Applications in medical data set, in: Data Analysis in Medicine and Pharmacology (IDAMAP- 2001), London, UK, 2001.

[23] D. Dheeru, E. Karra Taniskidou, UCI machine learning repository, Available: https://archive.ics.uci.edu/ml/datasets/SCADI, 2017. [Dataset].

[24] W. S. McCulloch, W. Pitts, A logical calculus of the ideas immanent in nervous activity, The bulletin of mathematical biophysics 5 (1943) 115-133.

[25] F. Rosenblatt, Principles of Neurodynamics: Perceptrons and the Theory of Brain Mechanisms, Spartan Books, Washington, 1962.

[26] D. E. Rumelhart, G. E. Hinton, R. J. Williams, Learning internal representations by error propagation, in: D. E. Rumelhart, J. L. McClelland, C. PDP Research Group (Eds.), Parallel Distributed Processing: Explorations in the Microstructure of Cognition, Vol. 1, MIT Press, Cambridge, MA, USA, 1986, pp. $318-362$.

[27] F. Rosenblatt, The perceptron: A probabilistic model for information storage and organization in the brain, Psychological Review 65 (1958) 386-408.

[28] B. Cheng, D. M. Titterington, Neural networks: A review from a statistical perspective, Statistical Science 9 (1994) 2-30.

[29] C. M. Bishop, Pattern Recognition and Machine Learning (Information Science and Statistics), Springer-Verlag, Berlin, Heidelberg, 2006.

[30] S. Putatunda, Machine Learning: An Introduction, in: A. K. Laha (Ed.), Advances in Analytics and Applications, Springer Proceedings in Business and Economics, Springer Nature Singapore Pte Ltd., 2019, pp. 1-9. doi:https: //doi.org/10.1007/978-981-13-1208-3_1.

[31] J. Larsen, Introduction to Artificial Neural Network, 1st ed., Technical University of Denmark, 1999.

[32] A. Kumar, M. Singh, S. Ghosh, A. Anand, Weather forecasting model using artificial neural network, Procedia Technology 4 (2012) 311-318.

[33] W. G. Baxt, Application of neural networks to clinical medicine, Lancet 346 (1995) 1135-1138.

[34] M. H. Eng, Y. Li, Q.-G. Wang, T. H. Lee, Forecast forex with ann using fundamental data, in: 2008 International Conference on Information Management, Innovation Management and Industrial Engineering, IEEE, Taipei, Taiwan, 2008. doi $10.1109 /$ ICIII.2008.302.

[35] A. Laha, S. Putatunda, Real time location prediction with taxi-gps data streams, Transportation Research Part C: Emerging Technologies 92 (2018) 298-322. 
[36] A. K. Laha, S. Putatunda, Travel Time Prediction for GPS Taxi Data Streams, Indian Institute of Management Ahmedabad, Working Paper No. 2017-03-03 (2017).

[37] S. Putatunda, Streaming Data: New Models and Methods with Applications in the Transportation Industry, Ph.D. thesis, Indian Institute of Management Ahmedabad, 2017.

[38] C. M. Bishop, Neural Networks for Pattern Recognition, 0198538642, Oxford University Press, Inc., New York, NY, USA, 1995.

[39] I. Goodfellow, Y. Bengio, A. Courville, Deep Learning, MIT Press, 2016.

[40] F. Chollet, Deep Learning with Python, 1st ed., Manning Publications Co., 2017.

[41] J. Wang, H. He, D. V. Prokhorov, A folded neural network autoencoder for dimensionality reduction, Procedia Computer Science 13 (2012) 120-127.

[42] D. Ghosh, A. Olewnik, K. Lewis, Application of autoencoders in cyber-empathic design, Design Science 4 (2018).

[43] T. Blaschke, M. Olivecrona, O. Engkvist, J. Bajorath, H. Chen, Application of generative autoencoder in de novo molecular design, Molecular Informatics 37 (2018).

[44] L. Breiman, J. Friedman, C. J. Stone, R. Olshen, Classification and regression trees, Taylor \& Francis, 1984.

[45] W.-Y. Loh, Fifty years of classification and regression trees, International Statistical Review 82 (2014) 329-348.

[46] T. Chen, C. Guestrin, XGBoost: A Scalable Tree Boosting System, in: Proceedings of the 22nd ACM SIGKDD International Conference on Knowledge Discovery and Data Mining, KDD '16, ACM, New York, NY, USA, 2016, pp. 785-794.

[47] L. Breiman, Random forests, Machine Learning 45 (2001) 5-32. Kluwer Academic Publishers, Hingham, MA, USA.

[48] T. Cover, P. Hart, Nearest neighbor pattern classification, IEEE Trans. Inf. Theor. 13 (1967) 21-27.

[49] C. Cortes, V. Vapnik, Support-Vector Networks, Mach. Learn. 20 (1995) 273297.

[50] G. I. Webb, Naïve Bayes, Springer, Boston, MA, 2010, pp. 713-714.

[51] T. Hastie, R. Tibshirani, J. Friedman, The Elements of Statistical Learning, Springer series in statistics, 2nd ed., Springer, 2009. New York.

[52] G. James, D. Witten, T. Hastie, R. Tibshirani, An Introduction to Statistical Learning with Applications in R, Springer, 2013. 
[53] G. Rossum, Python Reference Manual, Technical Report, CWI (Centre for Mathematics and Computer Science), Amsterdam, The Netherlands, The Netherlands, 1995.

[54] F. Pedregosa, G. Varoquaux, A. Gramfort, V. Michel, B. Thirion, O. Grisel, M. Blondel, P. Prettenhofer, R. Weiss, V. Dubourg, J. Vanderplas, A. Passos, D. Cournapeau, M. Brucher, M. Perrot, E. Duchesnay, Scikit-learn: Machine Learning in Python, Journal of Machine Learning Research 12 (2011) 28252830.

[55] M. Abadi, P. Barham, J. Chen, Z. Chen, A. Davis, J. Dean, M. Devin, S. Ghemawat, G. Irving, M. Isard, M. Kudlur, J. Levenberg, R. Monga, S. Moore, D. G. Murray, B. Steiner, P. Tucker, V. Vasudevan, P. Warden, M. Wicke, Y. Yu, $\mathrm{X}$. Zheng, Tensorflow: A system for large-scale machine learning, in: Proceedings of the 12th USENIX Conference on Operating Systems Design and Implementation, USENIX Association, Berkeley, CA, USA, 2016, pp. 265-283.

[56] Chollet et al., Keras, https://keras.io, 2015.

[57] P. Refaeilzadeh, L. Tang, H. Liu, Cross-validation, in: L. Liu, M. Özsu (Eds.), Encyclopedia of Database Systems, Springer, Boston, MA, 2009.

[58] N. Srivastava, G. Hinton, A. Krizhevsky, I. Sutskever, R. Salakhutdinov, Dropout: A Simple Way to Prevent Neural Networks from Overfitting, Journal of Machine Learning Research 15 (2014) 1929-1958. 